\title{
Tension Band Wiring of the Olecranon: A Biomechanical Study
}

\author{
Jose Ma D. Bautista and Rene Edgardo C. Manalastas \\ Department of Orthopedics, College of Medicine and Philippine General Hospital, University of the Philippines Manila
}

\begin{abstract}
Objective. Hardware prominence is the most common cause of reoperation in patients who have undergone tension band wiring for fractures of the olecranon. The study was conducted to compare the strength of four different constructs of tension band wiring of the olecranon.

Methods. A transverse fracture was created in twenty cadaveric ulnas which were equally divided into four groups according to the method of fixation: 1-Single tension band, transcortical kwire fixation; 2-Single tension band, intramedullary k-wire fixation; 3-Double tension band, transcortical k-wire fixation; 4Double tension band, intramedullary k-wire fixation. All specimens were mounted on a universal testing machine using a custom-made fixation jig. They were tested under a single cycle load to failure. The maximum failure load was measured for all specimens.
\end{abstract}

Results. There was no significant difference in the maximum load to failure among the four treatment groups.

Conclusion. No differences in the maximum load to failure were noted in between treatment groups, regardless if single or double tension band wiring was done, and regardless if k-wire fixation was transcortical or medullary. These findings would support the argument that any of the desired fixation methods may be used.

Key Words: olecranon fracture, tension band wiring, biomechanical study

\section{Introduction}

Olecranon fractures occur in a bimodal distribution, usually sustained as high energy injuries in the young and as low energy falls in elderly patients. The usual mechanism of injury is a direct blow to the elbow resulting in a comminuted fracture, or from an indirect blow such as a fall onto an outstretched hand, resulting in a transverse or oblique fracture.

Tension band wiring is generally accepted as the standard of care for treatment of displaced olecranon

Corresponding Author: Jose Ma D. Bautista, MD

Department of Orthopedics

Philippine General Hospital

University of the Philippines Manila

Taft Avenue, Ermita, Manila 1000 Philippines

Telephone: +6325548466

Fax No.:+ 6325218538

Email: bautistajosema@gmail.com fractures. This construct is designed to convert tensile distraction forces from the triceps into a compressive force over the articular surface. Common complications include infection, implant failure, pin migration, and prominence of hardware, which is the most commonly reported complication. ${ }^{1,2}$

Prominence of hardware is due to the use of a relatively thick wire placed in a relatively superficial location, which can lead to other complications such as skin necrosis and infection. In order to minimize hardware prominence, Kim and his colleagues ${ }^{3}$ have described a technique wherein they use thinner wires in a double tension band configuration, with promising results. They have done this procedure in 21 patients with a mean follow up of 37 months, with results showing no loss of reduction and no non-union or migration of the k-wires. Average flexion and extension arc was at 132 degrees, and hardware removal was done for 13 cases $(62 \%)$. Biomechanical testing was also done. They found that mean maximum failure load and mean maximum bending moments at failure were significantly higher in the double tension band wiring group as compared to the single tension band wiring group.

Symptomatic prominence is a frequent complication, resulting in a high rate of reoperations for implant removal. ${ }^{4,5}$ The use of transcortical k-wire fixation has been proposed to reduce the incidence of pin migration and pullout. Conflicting results have been published regarding the configuration of the anchoring $\mathrm{k}$-wires for tension band wiring techniques. Kuo, et al. showed that using bicortical kwire purchase significantly decreased the migration of the kwires as compared to intramedullary fixation. However, there may be a greater risk to the articular cartilage when attempting to aim for the anterior cortex of the ulna. ${ }^{6}$ Chan and Donnelly in 2014 have shown that there is no significant difference in the pullout or pin migration resulting in removal of the construct. ${ }^{4}$ Suter likewise showed no significant difference in pull-out strength when he compared different models of transcortical k-wire fixation: anteriorly and posteriorly directed. ${ }^{7}$ A local study by Dungca and Leagogo in 1992 showed that there was no observed separation of fragments in fractures fixed with transcortical k-wire fixation as compared to intramedullary k-wire fixation. ${ }^{8}$

In an effort to prevent wire migration after tension band fixation of olecranon fractures Larsen and Jensen used nonsliding pins which they designed. They had no outward pin 
migration in 20 patients and had good clinical outcomes. ${ }^{9}$ These pins however are not yet commercially available, nor were biomechanical tests performed.

No biomechanical studies have yet been done comparing the different combinations of k-wire fixation and tension band wiring.

The study aimed to compare the strength of four constructs using a combination of either a single or double tension band wire with either transcortical or intramedullary $\mathrm{k}$-wire fixation in terms of load to failure.

\section{Methods}

Twenty (20) ulnas were obtained for the study, and radiographs were taken to assess for uniformity of specimens in terms of bone quality. A transverse fracture through the middle of the trochlear notch of the ulna was made using a $0.5 \mathrm{~mm}$ saw blade. Once the fractures were made for all the ulnas, they were then equally distributed among the four different fixation constructs. In all study arms, the fracture will be reduced and held in place with reduction clamps prior to doing the tension band wiring.

\section{Single tension band, transcortical $k$-wire fixation}

A 0.062-inch Kirschner wire was inserted from the radial dorsal cortex of the proximal fragment, going obliquely towards the anterior cortex of the distal fragment of the ulna. A second Kirschner wire was inserted parallel to the first k-wire using a parallel-hole drill guide. Once the two k-wires were inserted, a drill hole was made $40 \mathrm{~mm}$ from the fracture line and $5 \mathrm{~mm}$ from the anterior cortex using a $2 \mathrm{~mm}$ drill. Tension band application was then applied following $\mathrm{AO}$ wiring techniques using Gauge 18 cerclage wire. Tightening of the loops of the tension band wire was then done manually (Figure 1).

\section{Single tension band, intramedullary $k$-wire fixation}

A 0.062-inch Kirschner was inserted to a length of $8 \mathrm{~cm}$ from the radial dorsal cortex of the proximal fragment, parallel to the longitudinal axis of the ulna. A second Kirschner wire was inserted parallel to the first k-wire using a parallel-hole drill guide. Once the two k-wires have been inserted, a drill hole was made $40 \mathrm{~mm}$ from the fracture line and $5 \mathrm{~mm}$ from the anterior cortex using a $2 \mathrm{~mm}$ drill. Tension band application was done following $\mathrm{AO}$ wiring techniques using Gauge 18 cerclage wire. Tightening of the loops of the tension band wire was then done manually (Figure 2).

\section{Double tension band, transcortical k-wire fixation}

A 0.062-inch Kirschner wire was inserted from the radial dorsal cortex of the proximal fragment, going obliquely towards the anterior cortex of the distal fragment of the ulna. A second Kirschner wire was inserted parallel to the first k-wire using a parallel-hole drill guide. Once the two k-wires have been inserted, a drill hole was made $40 \mathrm{~mm}$ from the fracture line and $5 \mathrm{~mm}$ from the anterior cortex using a $2 \mathrm{~mm}$ drill bit. Tension band application will then be applied following $\mathrm{AO}$ wiring techniques using Gauge 22 cerclage wire.

Another drill hole was made $20 \mathrm{~mm}$ from the fracture line and $5 \mathrm{~mm}$ from the anterior cortex using a $2 \mathrm{~mm}$ drill bit. Tension band application was done following $\mathrm{AO}$ wiring techniques using Gauge 22 cerclage wire. Tightening of the loops of the tension band wires was done manually (Figure 3).

Double tension band, intramedullary k-wire fixation

A 0.062-inch Kirschner wire was inserted from the radial dorsal cortex of the proximal fragment, parallel to the longitudinal axis of the ulna, to a depth of $8 \mathrm{~cm}$. A second Kirschner wire was inserted parallel to the first k-wire using a parallel-hole drill guide. Once the two k-wires have been inserted, a drill hole was made $40 \mathrm{~mm}$ from the fracture line and $5 \mathrm{~mm}$ from the anterior cortex using a $2 \mathrm{~mm}$ drill bit. Tension band application was done following AO wiring techniques using Gauge 22 cerclage wire. Tightening of the tension band was done manually.

Another drill hole was made $20 \mathrm{~mm}$ from the fracture line and $5 \mathrm{~mm}$ from the anterior cortex using a $2 \mathrm{~mm}$ drill bit. Tension band application was done following AO wiring techniques using Gauge 22 cerclage wire. Tightening of the tension band was done manually (Figure 4).

\section{Stress testing of the different constructs}

Each specimen was mounted on a universal testing machine (Shimadzu AutoGraph AGS-X Series) using a custom-made fixation jig to stabilize the proximal ulna, and to provide a counter-force against the pulling force on the gauge 16 cerclage wire which was looped through a drill hole in the proximal olecranon fragment through a hole $5 \mathrm{~mm}$ from the dorsal cortex and $5 \mathrm{~mm}$ from the proximal end of the fragment simulating the pull of the triceps on an elbow flexed at 90 degrees (Figure 5).

All specimens were tested under a single cycle load to failure at a displacement speed of $10-\mathrm{mm} / \mathrm{min}$. The maximum failure load, which is defined as the point at which the load-displacement curve abruptly decreases, was measured for all specimens.

An independent observer was present throughout the testing of the different constructs.

Analysis of variance (ANOVA) was done using StatPlus: Mac LE to determine if the results of the study were homogenous or heterogeneous, which would determine the type of t-test to be done. T-test was done comparing the load to failure of the different treatment groups using Microsoft Excel for Mac 2011. The level of significance was set at $\mathrm{p}<0.05$. 


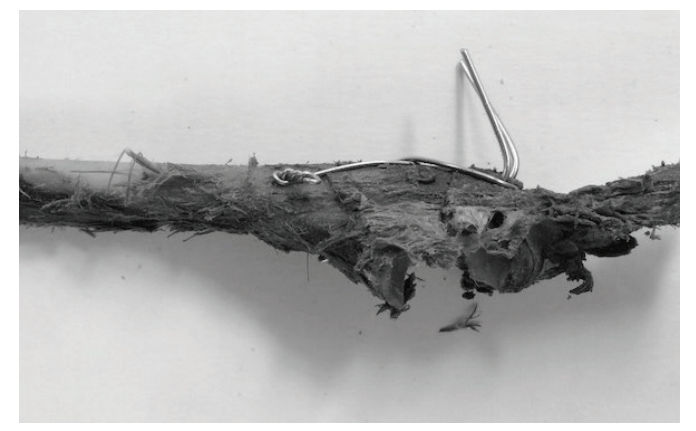

Figure 1. Single tension band wire, transcortical k-wire fixation.

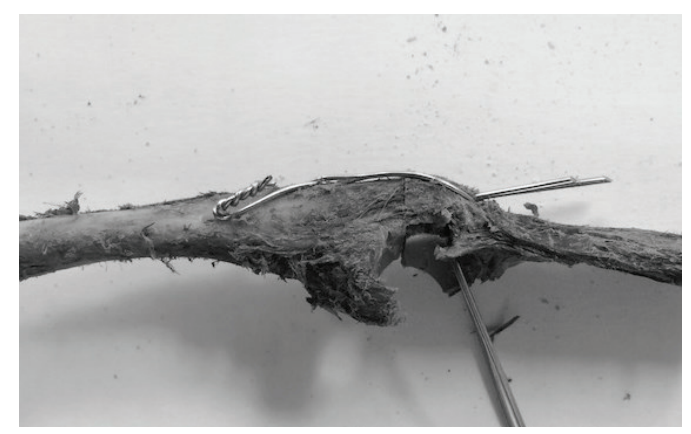

Figure 2. Single tension band wire, intramedullary k-wire fixation.

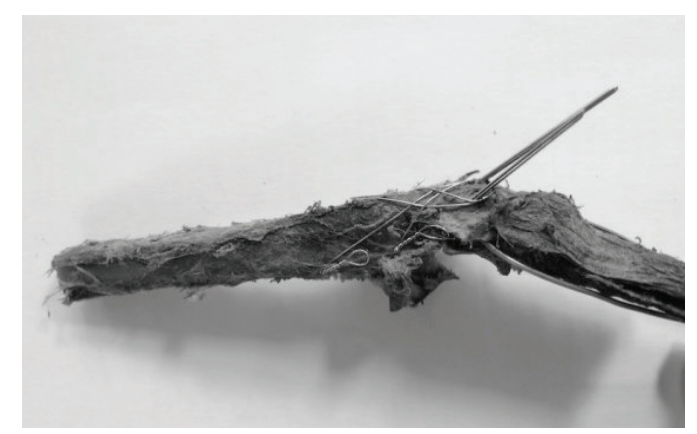

Figure 3. Double tension band, transcortical k-wire fixation.

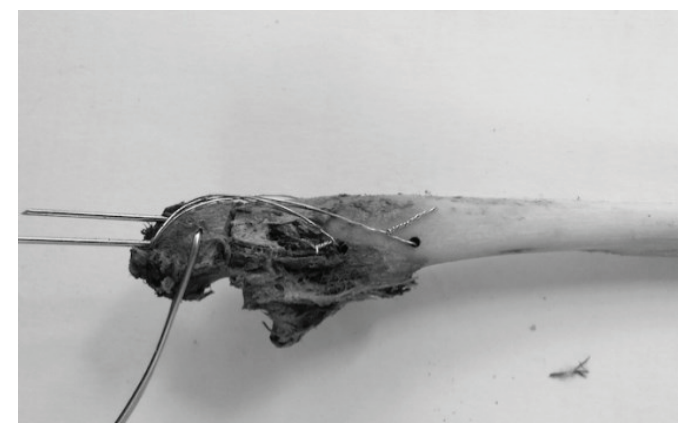

Figure 4. Double tension band, intramedullary k-wire fixation.

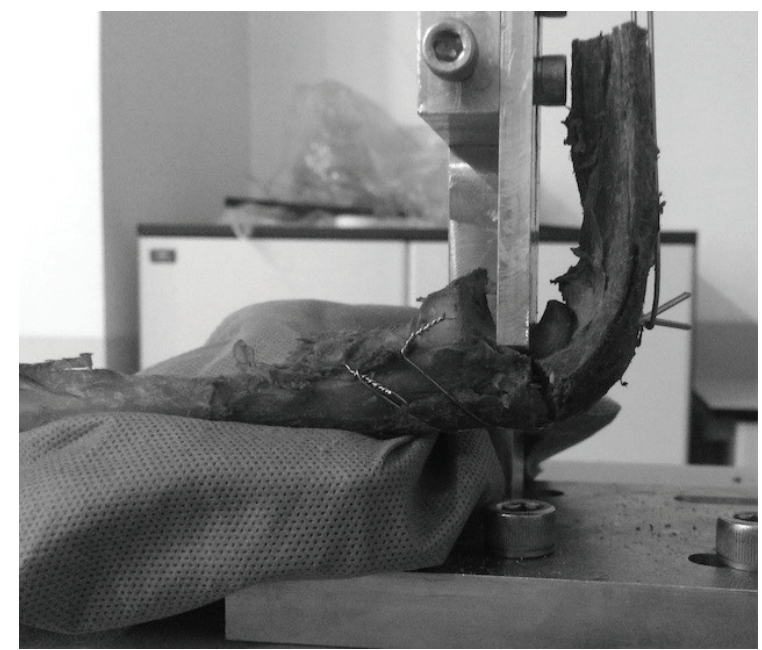

Figure 5. Experiment setup with a custom jig holding the elbow in a simulated 90-degree flexion. Wire is attached to the universal testing machine, simulating the triceps tendon.

\section{Results}

Most of the specimens tested (16 specimens) failed through the bone when the wire simulating the triceps cut through the cadaveric bone, resulting in an abrupt decrease in the load-displacement curve, with the constructs remaining intact. In the first and third treatment groups (transcortical K-wire fixation), all specimens tested resulted in failure through the bone. Gapping of the posterior cortex was also observed, although the continuity of the anterior cortex remained intact. In the second and fourth treatment groups (intramedullary K-wire fixation), there were two incidences wherein a step-off deformity of the fracture site developed. In these instances, the intramedullary k-wires were bent through the fracture site. There was no note of any pullout of wires in the first and third treatment groups (transcortical K-wire fixation).

For the single tension band, transcortical fixation group, values ranged from 529.56 to 1217.49 Newtons, with a mean value of $690.52 \mathrm{~N}$. For the second treatment group, values

Table 1. Maximum Load to Failure Measured in Newtons

\begin{tabular}{ccccc}
\hline Specimen number & Group 1 & Group 2 & Group 3 & Group 4 \\
\hline 1 & 529.56 & 1057.54 & 487.63 & 227.54 \\
2 & 497.56 & 633.97 & 348.85 & 534.29 \\
3 & 592.33 & 827.67 & 604.13 & 610.54 \\
4 & 1217.49 & 682.88 & 462.41 & 725.02 \\
5 & 615.67 & 558.12 & 1236.32 & 568.46 \\
Mean & 690.522 & 752.036 & 627.868 & 533.17 \\
\hline
\end{tabular}

Group 1 is the single tension band wire using G18 cerclage wire, with transcortical k-wire fixation

Group 2 is the single tension band wire using G18 cerclage wire, with intramedullary $k$-wire fixation

Group 3 is the double tension band wire using G22 cerclage wire, with transcortical $k$-wire fixation

Group 4 is the double tension band wire using G22 cerclage wire, with intramedullary $k$-wire fixation 
Table 2. ANOVA Results. ( $\mathrm{F}<\mathrm{F}_{\text {crit, }}$ indicating equal variance between the treatment groups).

Analysis of Variance (One-Way)

\begin{tabular}{|c|c|c|c|c|c|c|}
\hline Groups & Sample size & Sum & Mean & Variance & & \\
\hline Group 1 & 5 & $3,452.61$ & 690.522 & $89,020.71567$ & & \\
\hline Group 2 & 5 & $3,760.18$ & 752.036 & $38,844.68593$ & & \\
\hline Group 3 & 5 & $3,139.34$ & 627.868 & $123,917.85492$ & & \\
\hline Group 4 & 5 & $2,665.85$ & 533.17 & $34,362.2187$ & & \\
\hline \multicolumn{7}{|l|}{ ANOVA } \\
\hline Source of Variation & SS & $\mathrm{df}$ & MS & $\mathbf{F}$ & p-level & $F_{\text {crit }}$ \\
\hline Between Groups & $130,946.0965$ & 3 & $43,648.69883$ & 0.61016 & $61.81089 \%$ & 3.23887 \\
\hline Within Groups & $1,144,581.90088$ & 16 & $71,536.36881$ & & & \\
\hline Total & $1,275,527.99738$ & 19 & & & & \\
\hline
\end{tabular}

ranged from 558.12 to 1057.54 , with a mean value of 752.04 N. For the third treatment group, values ranged from 348.85 to $1236.32 \mathrm{~N}$, with a mean of $627.87 \mathrm{~N}$, while for the fourth treatment group, values ranged from 227.54 to $725.02 \mathrm{~N}$, with a mean value of $533.17 \mathrm{~N}$ (Table 1). Results showed that $\mathrm{F}<\mathrm{F}_{\text {crit, }}$ indicating that a paired t-test can be done for the study (Table 2). The paired two-tailed T-test showed no significant differences between the first treatment group and the other treatment arms (Table 3).

There was no significant difference in the maximum load to failure when comparing single and double tension band wiring, regardless if it was for the transcortical or intramedullary single tension band. We also found that there was no significant difference in the maximum load to failure for the third and fourth treatment group (Table 3).

\section{Discussion}

Given the high rate of symptomatic prominence of the implants associated with tension band wiring of fractures of the olecranon, any less prominent but biomechanically equivalent modification of the technique must be considered.

The transcortical application of the K-wires has been reported to result in less pull-out. ${ }^{6,8}$ The use of two tension band wires (using thinner gauge materials) has likewise been proposed to reduce hardware prominence. ${ }^{3}$

In this study, where four different combination of olecranon tension band wiring was done, the maximum load to failure was recorded as the point where there is an abrupt decrease in the load-displacement curve. The investigators and another independent observer observed this during the testing. Most of the specimens tested failed through the bone when the wire simulating the triceps cut through the cadaveric bone, resulting in the abrupt decrease, with the constructs remaining intact. There were also incidences wherein the Kirschner wires would cut out through the dorsal cortex of the proximal fragment. This may be due to the nature of the cadaveric bone, which may have relative osteopenia compared to normal bone. However, in the study by Dungca and Leagogo in 1992, they cited that cadaveric
Table 3. p-values for Homoscedastic Two-tailed T-test $(p<0.05)$ Comparing the Different Treatment Groups

\begin{tabular}{lc}
\hline Group 1 vs Group 2 & 0.75 \\
Group 1 vs Group 3 & 0.79 \\
Group 1 vs Group 4 & 0.20 \\
Group 2 vs Group 3 & 0.59 \\
Group 2 vs Group 4 & 0.24 \\
Group 3 vs Group 4 & 0.61 \\
\hline Group 1 is the single tension band wire using G18 cerclage wire, with transcortical \\
k-wire fixation \\
Group 2 is the single tension band wire using G18 cerclage wire, with \\
intramedullary $k$-wire fixation \\
Group 3 is the double tension band wire using G22 cerclage wire, with transcortical \\
k-wire fixation \\
Group 4 is the double tension band wire using G22 cerclage wire, with \\
intramedullary $k$-wire fixation
\end{tabular}
intramedullary $k$-wire fixation

bone had similar strengths to normal bone. ${ }^{8}$ The concentration of forces through the proximal fragment without any counteracting forces may have contributed to the cutout as well. To our knowledge, no other study mentioned the cutout through bone, although gapping or compression of the anterior cortex was mentioned in the study by Dungca and Leagogo. In the study by Kim et al., they mentioned that the failure of the construct occurred through the osteotomy site, although this was not further elaborated. ${ }^{3}$ Since testing was done for maximum load to failure, we would not expect to see the same type of failure if these fixation methods were used in vivo, since there would be a natural tendency towards protective actions.

Results of the study show that there were no significant differences in the mean maximum load to failure for the four treatment arms. This is consistent with the results of the study of Chan and Donnelly which showed no significant difference in the pullout or pin migration with either transcortical or intramedullary pin fixation. ${ }^{4}$

For the first treatment arm, all specimens resulted failure through the bone. There was also gapping of the posterior cortex, although the continuity of the anterior cortex was maintained. Results and outcomes were similar for the third treatment arm. Similar to the findings in a study by Kuo, et al. in 2011, there was no note of pull out or migration of the k-wires in the transcortical groups. ${ }^{6}$ 
For the second and fourth treatment arms, there were two incidences wherein a step-off deformity of the fracture site developed. On further inspection, the intramedullary kwires were bent through the fracture site. One possible explanation may be that the fulcrum could have been placed slightly more anterior to the fracture site, resulting in a direct upward pull on the proximal fragment. There was also a greater incidence of migration or pullout of the kwires, although these were based on inspection only and not quantified. The greater incidence of migration mirrors the findings of Mullet and his colleagues in 2000, wherein they found that fractures fixed with intramedullary k-wire configuration had three times the rate of pins backing out compared to transcortical fixation. ${ }^{10}$

To further simulate the daily wear of motion on the construct, cyclic load testing could have been done. However, this was not done due to limitations in the availability of a machine capable of doing a cyclic load testing. The tensile force could have likewise been applied on the triceps tendon rather than on the inserted gauge 16 wire. However, as the cadavers were not fresh, the tendons could not hold the suture wires when tension was applied.

In line with the findings of this study, we can suggest that a prospective randomized controlled trial can be done to compare the clinical outcomes of hardware prominence, rate of removal, pin migration, and complication rates for the different constructs.

\section{Conclusion}

No differences in the maximum load to failure were noted in between treatment groups, regardless if single or double tension band wiring was done, and regardless if $\mathrm{k}-$ wire fixation was transcortical or intramedullary. These findings would support the argument that any of the desired fixation methods can be used without jeopardizing the strength of the construct and would allow for early range of motion, the primary advantage for using tension band wiring in fixing olecranon fractures.

\section{Acknowledgements}

The authors thank Dr. Rafael Bundoc for advice regarding the biomechanical analysis and Dr. Emmanuel Estrella for reviewing the manuscript.

\section{References}

1. Chalidis BE, Sachinis NC, Samoladas EP, Dimitriou CG, Pournaras JD. Is tension band wiring technique the "gold standard" for the treatment of olecranon fractures? A long term functional outcome study. J Orthop Surg Res. 2008; 3:9.

2. Davies MB, King CK, Stanley D. Tension band wiring of olecranon fractures. Shoulder Elbow. 2009; 1(1):51-4.

3. Kim W, Choi S, Yoon JO, Park HY, Kim SH, Kim JS. Double tension band wiring for the treatment of olecranon fractures. J Hand Surg Am. 2014; 39(12):2438-43.

4. Chan KW, Donnelly KJ. Does K-wire position in tension band wiring of olecranon fractures affect its complications and removal of metal rate? J Orthop. 2014; 12(2):111-7.

5. Macko D, Szabo RM. Complications of tension-band wiring of olecranon fractures. J Bone Joint Surg Am. 1985; 67(9):1396-401.

6. Kuo CC, Hsu HC, Hong SW, Al E. Biomechanical assessment of tension band wiring for olecranon fractures. Biomed Eng - App Bas C. 2011; 23:83-7.

7. Suter P. Tension-band wiring of olecranon fractures - Biomechanical analysis of different fixation techniques. Mémoire de Maîtrise en medicine. 2012 No 908.

8. Dungca GV, Leagogo LAC. Tension band wiring techniques for transverse olecranon fractures: an experimental mechanical study. Philippine Journal of Orthopedics. 1992; 11(1):40-3.

9. Larsen E, Jenssen CM. Tension-band wiring of olecranon fractures with non-sliding pins. Acta Orthop Scand. 1991; 62(4):360-2.

10. Mullet JH, Shannon F, Noel J, Lawlor G, Lee TC, O'Rourke SK. K-wire position in tension band wiring of the olecranon - a comparison of two techniques. Injury. 2000; 31(6):427-31. 